\title{
Motion Planning for Human-Robot Collaboration Using an Objective-Switching Strategy
}

\author{
Akira Kanazawa, Member, IEEE, Jun Kinugawa, Member, IEEE, and Kazuhiro Kosuge, Fellow, IEEE
}

\begin{abstract}
Motion planning of collaborative robots is often required to simultaneously satisfy the contradictory objectives of reliably avoiding human workers and safely approaching them. In this paper, we propose a new strategy that adaptively selects one of two objective functions based on the current operational region of the robot; the objective function for avoiding the worker with a distance larger than the safe distance, and the objective function for approaching the worker with a speed limitation for human safety. This strategy improves worker safety while limiting the negative impact of the speed limit on timeefficiency. The chattering related to the switching between the two objectives is solved using a continuous approximation of the switching based on uncertainties of the predicted worker's motion. We implement the proposed motion planning with the objective-switching strategy into a collaborative assembly system to deal with the worker moving with high irregularities. We experimentally evaluate the effectiveness of the proposed motion planning from the safety and efficiency points of view.
\end{abstract}

Index Terms-Collaborative Robot, Cognitive Human-Robot Interaction, Motion Planning, Learning and Adaptive Systems

\section{INTRODUCTION}

$\mathbf{H}$ UMAn-robot collaborative systems, in which a robot shares its workspace with human workers and works in cooperation with them, are becoming common in many factories. The introduction of the human-robot collaborative system is expected to combine the advantages of automation with the skills of human workers and makes it possible to build more compact and flexible production lines [1]. Ensuring worker safety while maintaining improved productivity by the robots is the most important problem in the design of such collaborative systems. Motion planning for collision avoidance is one of the most common and direct solutions to ensure the safety of workers of the human-robot collaborative systems. This paper focuses on both the safety and the efficiency of the collaborative robot systems from the motion planning point of view.

To plan the motion of the collaborative robots, we must simultaneously consider reliably avoiding human workers and safely approaching them. Although worker safety should be the top priority, collaborative robots often need to approach the workers to support their tasks, increasing the risk of collision. Such a complex situation is difficult to be solved by simple motion planning for collision avoidance with human workers.

As a guideline for robots to safely operate in human-robot collaborative environments, ISO 10218-1 has identified four

A. Kanazawa, J. Kinugawa and K. Kosuge are with the Department of Robotics, Tohoku University, 6-6-01, AzaAoba, Aramaki, Aoba-ku, Sendai-shi, Miyagi-ken, 980-8579, Japan. (email: \{kanazawa, kinugawa, kosuge\}@irs.mech.tohoku.ac.jp) collaborative modes, including 1) safety-rated monitored stop, 2) hand guiding, 3) speed and separation monitoring, and 4) power and force limited [2]. To achieve both safety and efficiency of a collaborative system that requires physical interaction between a robot and workers, the motion planning of the collaborative robot must take requirement 3) into account.

To meet requirement 3 ), the robot is required to maintain a predetermined safe speed and distance from the worker during operation. Many studies have addressed this issue and have proposed methods to plan the robot motion that reduces the speed of the robot adaptively and reasonably in close proximity to the workers [18][19][20]. These methods have made significant contributions to safe collaboration between workers and robots, but further improvement in time-efficiency is still necessary.

In this paper, we propose a motion planning strategy that explicitly incorporates a safe approach to the worker and the avoidance of collision with the worker. The proposed algorithm switches the objective functions for the robot's motion depending on the region where the robot is currently operating. The contributions of this paper are summarized as follows;

1) The proposed motion planner is an optimization-based motion planner, which dynamically switches between the two objective functions. One is the objective function for avoiding the worker and the other is the objective function for approaching the worker.

2) The proposed motion planner increases the success rate of the motion planning for approaching the worker while improving safety, and time efficiency of the collaborative work compared to simply stopping or slowing down the robot motion.

3) The switching-based strategy is combined with the prediction-based method proposed in our previous study [3]. This improves worker safety without affecting the efficiency of the prediction-based system.

4) The chattering inherent to the switching between the two objectives is solved using a continuous approximation of the switching based on uncertainties of the predicted worker's motion.

5) Experiments show that the proposed system can effectively cope with various worker movements and simultaneously improve worker safety and time-efficiency.

The remainder of this paper is organized as follows. Section II summarizes the related works on motion planning focusing on the human-robot collaborative scenario. Section III explains the overview of the proposed collaborative framework. This 
framework includes safe approaching with constraints based on human motion prediction. Section IV explains the details of the optimization-based motion planner in which the objective-switching strategy is integrated with the predictionbased strategy. Section V presents experiments performed on several risky situations and discusses the effectiveness of the proposed system on safety and time-efficiency. Finally, section VI concludes this paper.

\section{RELATED WORKS}

\section{A. Manipulator motion planning}

In motion planning for industrial robots, global path/trajectory planning is the mainstream approach. For an articulated manipulator that is commonly used in factory automation, random sampling-based approaches such as probabilistic roadmap (PRM)-based [4] and rapidly random tree (RRT)-based approaches [5] are well-known. These sampling-based approaches can calculate a collision-free path in high-dimensional space in real-time.

Another well-known method is the local optimization-based method that can take two objectives of collision avoidance and trajectory smoothness into account. Ratliff et al. used Covariant Hamiltonian Optimization for Motion Planning (CHOMP) for the nonlinear optimization problem with the two objectives [6]. Kalakrishnan et al. proposed Stochastic Trajectory Optimization for Motion Planning (STOMP) to deal with collision check in the probabilistic manner [7]. Park et al. proposed Incremental Trajectory Optimization for Motion Planning (ITOMP) for real-time optimization of dynamic collision avoidance [8]. Schulman et al. used the sequential convex optimization to efficiently calculate the avoidance trajectory [9]. These methods can rapidly and effectively find a local optimal solution taking collision avoidance and trajectory smoothness into account.

With improvements in computational power, several global optimization methods have been proposed to solve the nonconvex problem. Kim et. al. proposed a motion planning method that satisfies multiple constraints using particle swarm optimization [10]. In recent years, Bayesian optimization was applied to motion planning of UAVs [11] and locomotion [12].

These global optimization techniques for non-convex problems with multiple objectives can derive better solutions, but they still require considerable computation power. Random sampling-based methods face difficulties in concurrently dealing with various objectives and constraints. In this paper, we propose a local optimization-based planning method to satisfy multiple objectives simultaneously in real-time.

\section{B. Planning motion in close proximity to human}

With the introduction of collaborative robots in recent years, motion planning strategies that allow robots to safely operate in close proximity to humans have become an important issue. Adaptive re-planning of the motion of robots depending on any unpredictable, irregular human movements is a key technique for addressing this problem. Hyne et al. proposed a signed distance field-based approach for dynamic collision avoidance with human [13]. Chen et al. applied a potential field-based approach to the collision-free motion planning [14]. Ceriani et al. proposed a collision-free collaborative system by effectively selecting the joint of a redundant manipulator [15]. Liu et al. discussed an algorithmic safety measure and summarizes a desirable controller structure in a collaborative scenario [16]. Kuli'c et al. proposed a planning strategy based on the multiple danger criteria including the human-robot relative distance and the robot inertia [17].

Another key technique is to limit the operational power and speed of robots, particularly their tool center point (TCP) near the human workers. Rojas et al. proposed a minimum jerk planner that limited the TCP velocity [18]. Weitschat et al. proposed a time-optimal motion generator that satisfies the speed limitation of the robot [19]. Zanchettin et al. introduced a control strategy that adjusts the robot's velocity depending on the human-robot relative distance [20]. Our proposed approach addresses adaptive avoidance and speed limitation in one planning. In particular, one of the contributions of this paper is to propose a planning framework for improving time efficiency.

\section{Planning based on human motion prediction}

In recent years, many studies have considered the effective use of human motion prediction in motion planning. One of their main objectives is to generate a safe trajectory using predicted human positions and their uncertainties. Mainprice et al. proposed a collision-free motion planner based on the predicted human occupancy [21]. Fridovich-Keil et al. used confidence-aware human motion prediction [22]. Park et al. presented an intent-aware motion planner that was based on the predicted worker actions with uncertainties [23]. Wang et al. proposed a trajectory generator using the predicted worker's skeleton motion [24].

Several studies have shown that human motion prediction is useful to facilitate fluent collaborative work and improve time-efficiency. Unhelkar et al. improved the time-efficiency by reducing the number and duration of robot stops based on human motion prediction [25]. Hawkins et al. proposed a wait-sensitive planner using a model matching-based predictor [26]. Maeda et al. proposed a smooth human-robot handover framework according to the estimated human motion phase [27]. Pérez-D'Arpino et. al. demonstrated collaborative work by classifying human reaching motions into several patterns from the obtained time-series data [28].

Our method incorporates an objective-switching strategy into the prediction-based motion planner proposed in our previous study [3]. This provides a significant increase in safety over the conventional system in [3] while maintaining time-efficiency improved by the prediction.

\section{PROPOSED MOTION PLANNING ARCHITECTURE}

A. Robot workspace, uninvadable region, and collaborative region

In this paper, we consider the motion planning of collaborative robots that help human workers performing some tasks by providing them with necessary objects. To plan the robot motion with the two contradictory requirements of avoiding 

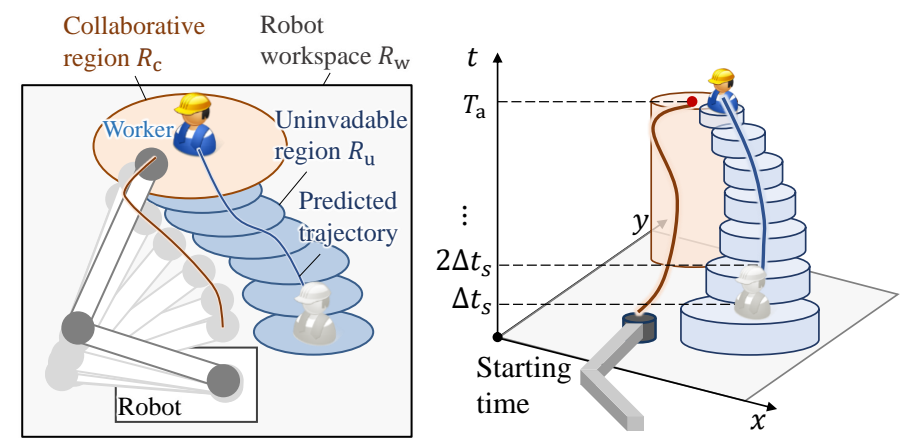

(a) Robot workspace, uninvad- (b) Motion planning in work-time able region, and collaborative re- space gion

Fig. 1: Concept of proposed motion planning

the human workers and approaching the human workers, we introduce a region-based switching strategy. Fig. 1 shows the concept of the proposed strategy based on three regions: robot workspace $R_{\mathrm{w}}$, uninvadable region $R_{\mathrm{u}}$, and collaborative region $R_{\mathrm{c}}$. Fig. 1 (a) illustrates $R_{\mathrm{w}}, R_{\mathrm{u}}$, and $R_{\mathrm{c}}$ in a planar workspace with two degrees of freedom manipulator. Although for simplicity of discussion, we focus on the $2 \mathrm{D}$ case with one worker as the receiver of the delivered objects, our proposed motion planning can be easily extended to the 3D workspace with multiple workers.

The robot workspace $R_{\mathrm{w}}\left(R_{\mathrm{w}} \subset \mathbb{R}^{2}\right)$ is the region that the robot, including its entire arm, moves and occupies without any restrictions. The uninvadable region $R_{\mathrm{u}}\left(R_{\mathrm{u}} \subseteq R_{\mathrm{w}}\right)$ is the region which the robot is not allowed to enter. This region is defined according to the workers' positions observed by a sensing system or predicted using the observed workers' movements. The collaborative region $R_{\mathrm{c}}\left(R_{\mathrm{c}} \subseteq R_{\mathrm{w}}\right)$ is the region in which the robot can enter and approach the worker to support the worker. This region is defined according to the working positions at which the workers are expected to work.

According to ISO 10218-1 [2], the safe approach to the worker in the collaborative workspace $R_{\mathrm{c}}$ is realized by approaching with a predetermined safe speed that is determined by a preliminary risk assessment. The robot can enter this region when its TCP speed is below the safe speed, that is, when the robot's kinetic energy is sufficiently small. In addition, in the collaborative workspace $R_{\mathrm{c}}$, the robot is required to immediately stop when the distance between the worker and the robot is less than a certain distance.

Fig. 1 (b) shows the proposed planning in the work-time space. The robot's trajectory is planned to arrive at the goal configuration at the desired arrival time. The predicted arrival time of the worker $T_{\mathrm{a}}$ is used for the desired arrival time of the robot so that an efficient motion with zero or small waiting time for the worker is expected. In motion planning, the robot must satisfy both the constraint of the uninvadable region $R_{\mathrm{u}}$ and the collaborative region $R_{\mathrm{c}}$. In the work-time space, $R_{\mathrm{u}}$ is predicted along the time axis at each sampling time based on the sensor measurement $\Delta t_{s}$, and $R_{\mathrm{c}}$ is defined around the working position along the time axis. We note that if the collaborative region $R_{\mathrm{c}}$ and the uninvadable region
$R_{\mathrm{u}}$ overlap, the constraints of the collaborative region $R_{\mathrm{c}}$ take precedence. In other words, the safety of the worker is enhanced by reducing the robot speed rather than by avoiding the worker in the vicinity of the working position.

\section{B. Worker motion prediction}

A mixture-model-based prediction system proposed in our previous study [29] is used for the worker motion prediction. The proposed system constructs a working model including the spatial and temporal features of each worker using the Gaussian mixture model (GMM). Only an overview of the methods is given in this section. For the details of the method, please refer to our previous research [29].

In our system, a worker model represented by the GMM is used to estimate the working position and predict the trajectory of the worker's movement. The input of the working model is the $d$-th order position time series $\boldsymbol{x}_{\mathrm{s}}^{(k)}=$ $\left(\boldsymbol{x}_{\mathrm{w}}^{(k)} \boldsymbol{x}_{\mathrm{w}}^{(k-1)} \cdots \boldsymbol{x}_{\mathrm{w}}^{(k-d+1)}\right)^{\mathrm{T}} \in \mathbb{R}^{m d}$, where $\boldsymbol{x}_{\mathrm{w}}^{(k)} \in \mathbb{R}^{m}$ is the measured $m$-dimensional worker's position vector at the $k$-th sampling time.

The worker model outputs two distributions: the estimated distribution of the working position $\mathcal{N}_{\mathrm{c}}$ and the predicted position distributions $\mathcal{N}_{\mathrm{u}} . \mathcal{N}_{\mathrm{c}}$ is a Gaussian distribution extracted from the GMM, and represents the mean and the variance of the worker's past working position. In the following, $\mathcal{N}_{\mathrm{c}}$ is treated as the collaborative region $R_{\mathrm{c}}$.

The worker's predicted position distributions $\mathcal{N}_{\mathrm{u}}$ is calculated by converting GMM to GMM/GMR using Bayes' theorem. This represents the mean and the variance of the future moving trajectory of the worker. Let the maximum prediction length be $T_{p}$, then, the worker's predicted position distributions can be expressed as $\mathcal{N}_{\mathrm{u}}=\left(\mathcal{N}_{\mathrm{u}}^{(k)}, \cdots, \mathcal{N}_{\mathrm{u}}^{\left(k+T_{\mathrm{p}}\right)}\right)$. In the following, $\mathcal{N}_{\mathrm{u}}$ is treated as the uninvadable region $R_{\mathrm{u}}$.

Based on the predicted trajectory $\mathcal{N}_{\mathrm{u}}$, the time at which the worker reaches the working position $\mathcal{N}_{\mathrm{c}}$ is predicted. In our system, the predicted arrival time $T_{\mathrm{a}}\left(T_{\mathrm{a}} \leq T_{\mathrm{p}}\right)$ is the time when a worker's predicted position distribution $\mathcal{N}_{\mathrm{u}}^{(k)}$ reaches the working position distribution $\mathcal{N}_{\mathrm{c}}$ for the first time.

\section{System architecture}

Fig. 2 shows the architecture of the proposed system comprising three parts: model updater, trajectory generator, and robot controller. In the model updater, the GMM is updated by the procedure described in [29] each time when all tasks are finished. The initial model is constructed based on a given work schedule. During the work, the worker position time series $\boldsymbol{x}_{\mathrm{s}}$ obtained from the sensing system is stored and used for the model update.

In the trajectory generator, the behavior of the worker is predicted using the position time series $\boldsymbol{x}_{\mathrm{s}}$ as an input. The working position distribution $\mathcal{N}_{\mathrm{c}}$, the sequence of the predicted position distributions $\mathcal{N}_{\mathrm{u}}$, and the predicted arrival time $T_{\mathrm{a}}$ are predicted in the worker motion predictor and input to the trajectory generator. The goal position is pre-designed based on the work schedule. Then, the trajectory generator calculates the trajectory $\boldsymbol{Q}=\left(\boldsymbol{q}^{(k)}, \cdots, \boldsymbol{q}^{\left(k+T_{\mathrm{a}}\right)}\right)$, where $\boldsymbol{q}^{(k)}$ is the 


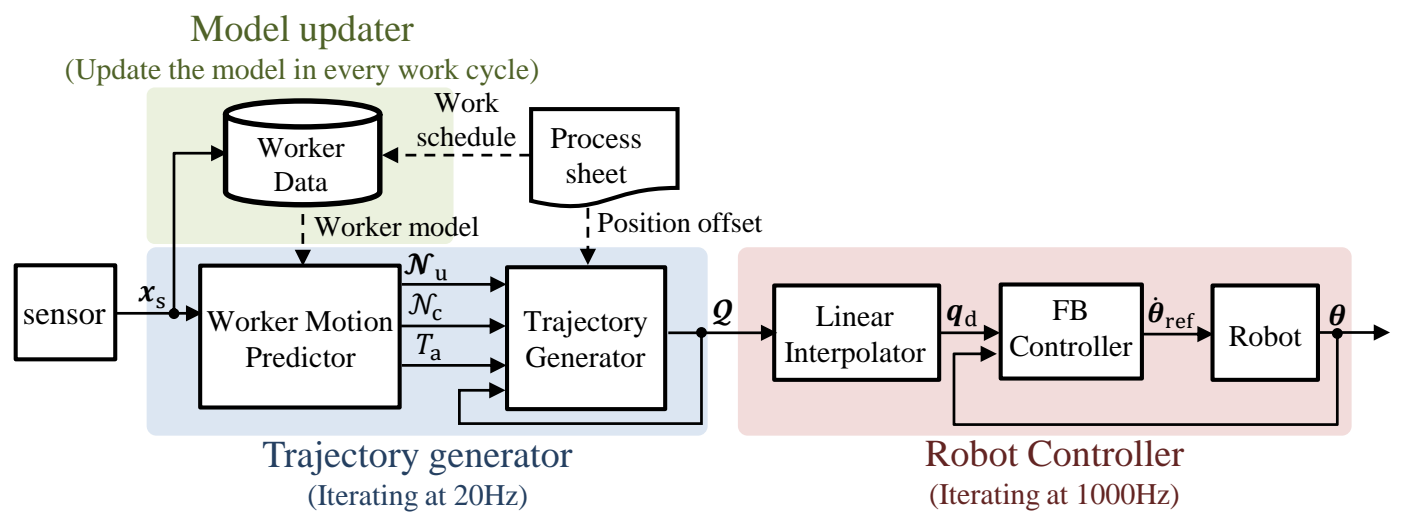

Fig. 2: System architecture.

robot's state at a $k$-th sampling time. In the proposed system, the robot trajectory $Q$ is calculated by solving the optimization problem that is described in detail in the next section. The trajectory generation is performed at every sampling time of the sensor measurement.

The optimized trajectory $Q$ calculated by the trajectory generator is linearly interpolated, and becomes the desired state of the robot $\boldsymbol{q}_{\mathrm{d}}$ that is input to the feedback controller whose sampling time is shorter than that of the trajectory generator. The feedback controller calculates the robot's joint velocity command $\dot{\boldsymbol{\theta}}_{\text {ref }}$, so that it can precisely follow the optimized trajectory $\boldsymbol{Q}$.

\section{OPTIMAL MOTION PLANNING WITH OBJECTIVE-SWITCHING STRATEGY}

\section{A. Problem formulation}

As briefly introduced in section III, we plan the robot motion with the two contradictory requirements of avoiding the human workers and approaching the human workers. We formulate the problem as an optimization problem with the objective-switching strategy according to where the current robot configuration is located, as follows:

$$
\begin{array}{ll}
\min & J=\varphi\left(\boldsymbol{q}\left(t_{0}+T_{\mathrm{a}}\right)\right)+\int_{t_{0}}^{t_{0}+T_{\mathrm{a}}} L(\boldsymbol{u}(t)) d t \\
\text { s.t. } & \dot{\boldsymbol{q}}(t)=f(\boldsymbol{q}(t), \boldsymbol{u}(t)) \\
& \boldsymbol{q}\left(t_{0}\right)=\boldsymbol{q}_{\mathrm{cur}} \\
& \left|\dot{q}(t)_{i}\right|<\dot{q}_{\max , i}\left(i=1, \cdots, 2 N_{j}\right) \\
& \boldsymbol{p}_{j}^{*}(\boldsymbol{q}(t)) \in R_{\mathrm{w}} \\
& \left\{\begin{array}{lll}
\boldsymbol{p}_{j}^{*}(\boldsymbol{q}(t)) \notin R_{\mathrm{u}} \quad(\text { if } & \left.\boldsymbol{p}_{j}^{*}(\boldsymbol{q}(t)) \notin R_{\mathrm{c}}\right) \\
\left|\boldsymbol{v}_{j}(\boldsymbol{q}(t))\right|<v_{\max } & (\text { if } & \left.\boldsymbol{p}_{j}^{*}(\boldsymbol{q}(t)) \in R_{\mathrm{c}}\right)
\end{array}\right.
\end{array}
$$

where $\boldsymbol{q}=(\boldsymbol{\theta}, \dot{\boldsymbol{\theta}})^{\mathrm{T}} \in \mathbb{R}^{2 N_{j}}$ is the state vector of the manipulator, $\boldsymbol{\theta}=\left(\theta_{1}, \theta_{2}, \cdots, \theta_{N_{j}}\right)^{\mathrm{T}} \in \mathbb{R}^{N_{j}}$ is the vector composed of the joint angles of the manipulator, $N_{j}$ is the motion degrees of freedom of the manipulator, $f$ represents the nonlinear term of the robot dynamics, $\boldsymbol{u} \in \mathbb{R}^{N_{j}}$ is the input vector for the robot, and $\boldsymbol{q}\left(t_{0}\right)$ is the initial state that corresponds to the robot current state $\boldsymbol{q}_{\text {cur }} \cdot \boldsymbol{x}_{j}=\left(\boldsymbol{p}_{j}, \boldsymbol{v}_{j}\right)^{\mathrm{T}}$ represents the pose and its velocity of the $j$-th joint in the workspace as shown in
Fig. 3. Note that $\boldsymbol{x}_{j}$ represents the pose of the joint coordinate system and its linear and angular velocity in the $n$-dimensional workspace $\left(n \leq N_{j}\right)$. $\boldsymbol{p}_{j}^{*}$ represents the position of every point on the $j$-th link segment. This is introduced to ensure that the whole manipulator satisfies the constraint defined according to the region.

The cost function $\varphi$ is defined as

$$
\begin{aligned}
& \varphi\left(\boldsymbol{q}\left(t_{0}+T_{\mathrm{a}}\right)\right) \\
& =\frac{1}{2}\left(\boldsymbol{x}_{N_{j}}\left(\boldsymbol{q}\left(t_{0}+T_{\mathrm{a}}\right)\right)-\boldsymbol{x}_{\mathrm{tgt}}\right)^{\mathrm{T}} S\left(\boldsymbol{x}_{N_{j}}\left(\boldsymbol{q}\left(t_{0}+T_{\mathrm{a}}\right)\right)-\boldsymbol{x}_{\mathrm{tgt}}\right) .
\end{aligned}
$$

This ensures the convergence of the endpoint state $\boldsymbol{x}_{N_{j}}$ to the neighborhood of the target state $\boldsymbol{x}_{\text {tgt }}$ at the final time $t_{0}+T_{\mathrm{a}}$. $\boldsymbol{x}_{\text {tgt }} \in \mathbb{R}^{2 n}$ represents the target pose and velocity of the robot endpoint in the $n$-dimensional workspace. $S \in \mathbb{R}^{2 n \times 2 n}$ is the diagonal positive definite weighting matrix.

The second term of eq. (1) is for suppressing the robot's input, and $L$ is expressed as

$$
L(\boldsymbol{u}(t))=\frac{1}{2} \boldsymbol{u}(t)^{\mathrm{T}} R \boldsymbol{u}(t),
$$

where $R \in \mathbb{R}^{N_{j} \times N_{j}}$ is the diagonal positive definite weighting matrix.

The equality constraints in eqs. (2) and (3) mean that the calculated trajectory is the output of the robot dynamics eq. (2) with the initial state $\boldsymbol{q}_{\text {cur }}$. The nonlinear term of the manipulator dynamics $f$ is expressed as

$$
\begin{aligned}
& f(\boldsymbol{q}(t), \boldsymbol{u}(t))= \\
& {\left[\begin{array}{c}
\dot{\boldsymbol{\theta}}(t) \\
\left.M^{-1}(\boldsymbol{\theta}(t))\left(\boldsymbol{u}(t)-\boldsymbol{c}(\boldsymbol{\theta}(t), \dot{\boldsymbol{\theta}}(t))-\boldsymbol{g}_{r}(\boldsymbol{\theta}(t))\right)\right],
\end{array}\right.}
\end{aligned}
$$

where $M$ is the inertia matrix, $\boldsymbol{c}$ is the Coriolis and Centrifugal term, and $\boldsymbol{g}_{r}$ is the gravity term. Eq. (4) is the inequality constraints ensuring that the angular velocity and acceleration of each joint are within their limit $\dot{q}_{\max , i}\left(i=1, \cdots, 2 N_{j}\right)$.

Eqs. (5) and (6) express the constraints related to the three regions: robot workspace $R_{\mathrm{w}}$, uninvadable region $R_{\mathrm{u}}$, and collaborative region $R_{\mathrm{c}}$. Eq. (5) is the constraint ensuring that the robot's link operates within the workspace $R_{\mathrm{w}}$. Eq. (6) is the constraint ensuring that the robot's link does not enter the uninvadable region $R_{\mathrm{u}}$ when it is outside of the collaborative region $R_{\mathrm{c}}$, and the maximum speed of the robot is reduced 


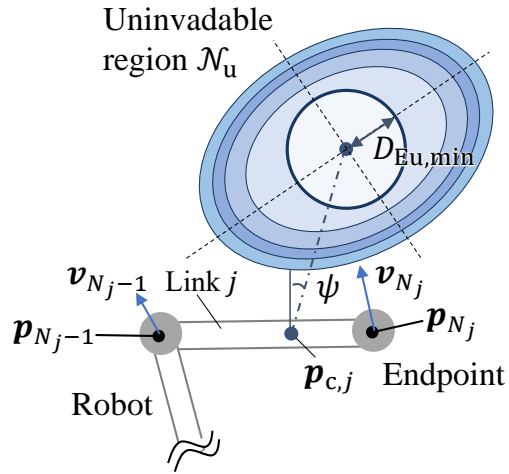

Fig. 3: Definition of the parameters of the robot and the uninvadable region.

to $v_{\max }$ when the robot is inside of the collaborative region $R_{\mathrm{c}}$. Each robot's link can enter the collaborative region $R_{\mathrm{c}}$ as long as its maximum speed is less than the threshold.

\section{B. Constraints in an analytical form}

The optimization problem formulated by eqs. (1) through (6) cannot be easily solved in its original form. To effectively deal with constraints including the differential equation, we use the calculus of variations [30][31]. The Euler-Lagrange equations that the optimal solution should satisfy are expressed as

$$
\begin{aligned}
& \dot{\boldsymbol{q}}(t)=f(\boldsymbol{q}(t), \boldsymbol{u}(t)), \\
& \boldsymbol{q}\left(t_{0}\right)=\boldsymbol{q}_{\mathrm{cur}}, \\
& \dot{\boldsymbol{\lambda}}(t)=-\left(\frac{\partial H}{\partial \boldsymbol{q}}\right)^{\mathrm{T}}(\boldsymbol{q}(t), \boldsymbol{u}(t), \boldsymbol{\lambda}(t)), \\
& \boldsymbol{\lambda}\left(t+T_{o}\right)=\left(\frac{\partial \varphi}{\partial \boldsymbol{q}}\right)^{\mathrm{T}}\left(\boldsymbol{q}\left(t_{0}+T_{\mathrm{a}}\right)\right), \\
& \frac{\partial H}{\partial \boldsymbol{u}}(\boldsymbol{q}(t), \boldsymbol{u}(t), \boldsymbol{\lambda}(t))=0
\end{aligned}
$$

where $\boldsymbol{\lambda} \in \mathbb{R}^{2 N_{j}}$ is the Lagrangian vector for the equality constraints. $H$ is the Hamiltonian for the inequality constraints and is represented as

$$
\begin{aligned}
& H(\boldsymbol{q}(t), \boldsymbol{u}(t), \boldsymbol{\lambda}(t)) \\
& =L(\boldsymbol{u}(t))+G(\boldsymbol{q}(t), \boldsymbol{u}(t))+\boldsymbol{\lambda}(\tau)^{\mathrm{T}} f(\boldsymbol{q}(t), \boldsymbol{u}(t)),
\end{aligned}
$$

where

$G(\boldsymbol{q}(t), \boldsymbol{u}(t))=\rho \sum_{j=1}^{N_{j}}\left(\sum_{c=1}^{N_{c}}\left|g_{c, j}(\boldsymbol{q}(t), \boldsymbol{u}(t))\right|^{+}+\left|g_{s, j}(\boldsymbol{q}(t))\right|^{+}\right)$

$\left|g_{c, j}\right|^{+}$and $\left|g_{s, j}\right|^{+}$are the L1 penalties, where $\left|g_{i, j}\right|^{+}=$ $\max \left(g_{i, j}, 0\right),(i=c, s) . \rho$ is the weight factor for the inequality constraints, and $N_{c}$ is the total number of the imposed inequality constraints $g_{c, j}$.
In the following part of this paper, we consider the 2D planar workspace for the simplicity of discussions. In the 2D planar workspace, $g_{c, j}$ are expressed as

$$
\begin{aligned}
& g_{1, j}(\boldsymbol{q}(t), \boldsymbol{u}(t))=p_{X \min }-p_{X c, j}(\boldsymbol{q}(t)) \\
& g_{2, j}(\boldsymbol{q}(t), \boldsymbol{u}(t))=p_{X c, j}(\boldsymbol{q}(t))-p_{X \max } \\
& g_{3, j}(\boldsymbol{q}(t), \boldsymbol{u}(t))=p_{Y \min }-p_{Y c, j}(\boldsymbol{q}(t)) \\
& g_{4, j}(\boldsymbol{q}(t), \boldsymbol{u}(t))=p_{Y c, j}(\boldsymbol{q}(t))-p_{Y \max } \\
& g_{5, j}(\boldsymbol{q}(t), \boldsymbol{u}(t))=\left|\dot{\theta}_{j}\right|-\dot{\theta}_{\max , j} \\
& g_{6, j}(\boldsymbol{q}(t), \boldsymbol{u}(t))=\left|\ddot{\theta}_{j}\right|-\ddot{\theta}_{\max , j},
\end{aligned}
$$

where $p_{X \min }, p_{X \max }, p_{Y \min }$, and $p_{Y \max }$ define the workspace $R_{\mathrm{w}} \cdot p_{X c, j}, p_{Y c, j}$ is the closest point to the workspace $R_{\mathrm{w}}$ among the points $\boldsymbol{p}_{j}^{*}$ on the link $j . \dot{\theta}_{\max , j}$ and $\ddot{\theta}_{\max , j}$ are the maximum angular velocity and acceleration for the joint $j$.

$g_{s, j}$ is the switching inequality constraint and is represented as

$$
g_{s, j}(\boldsymbol{q}(t))=\left\{\begin{array}{ll}
D_{\mathrm{th}, m}(\alpha)-D_{\mathrm{M}_{\mathrm{a}}, j}(\boldsymbol{q}(t)) & \text { if } \left.\boldsymbol{p}_{\mathrm{c}, j}^{*}(\boldsymbol{q}(t)) \notin R_{\mathrm{c}}\right) \\
\left|\boldsymbol{v}_{j}(\boldsymbol{q}(t))\right|-v_{\max } & \text { (if } \left.\boldsymbol{p}_{\mathrm{c}, j}^{*}(\boldsymbol{q}(t)) \in R_{\mathrm{c}}\right)
\end{array},\right.
$$

where $v_{\max }$ is the upper bound of the velocity $\boldsymbol{v}_{j}(\boldsymbol{q}(t))$ in the collaborative region. $D_{\mathrm{M}_{\mathrm{u}}, j}(\boldsymbol{q}(t))$ is expressed as

$$
D_{\mathrm{M}_{\mathrm{u}}, j}(\boldsymbol{q}(t))=D_{\mathrm{M}}\left(\left(\boldsymbol{p}_{\mathrm{c}, j}(\boldsymbol{q}(t))+\boldsymbol{D}_{\mathrm{E}_{\mathrm{u}}}\right), \boldsymbol{\mu}_{\mathrm{u}}^{(t)}, \boldsymbol{\Sigma}_{\mathrm{u}}^{(t)}\right),
$$

where

$$
\begin{aligned}
& \boldsymbol{p}_{\mathrm{c}, j}(\boldsymbol{q}(t))=\underset{\boldsymbol{p}_{j}^{*}}{\arg \min } D_{\mathrm{M}}\left(\boldsymbol{p}_{j}^{*}(\boldsymbol{q}(t)), \boldsymbol{\mu}_{\mathrm{u}}^{(t)}, \boldsymbol{\Sigma}_{\mathrm{u}}^{(t)}\right), \\
& \boldsymbol{D}_{\mathrm{E}_{\mathrm{u}}}=\left(D_{\mathrm{E}_{\mathrm{u}}, \min } \cos \psi, D_{\mathrm{E}_{\mathrm{u}}, \min } \sin \psi\right)^{\mathrm{T}},
\end{aligned}
$$

where $\boldsymbol{p}_{\mathrm{c}, j}$ is the closest point to the Gaussian distribution $\mathcal{N}_{\mathrm{u}}^{(t)}$ among the points $\boldsymbol{p}_{j}^{*}$ on the link $j . D_{\mathrm{M}}(\boldsymbol{x}, \boldsymbol{\mu}, \Sigma)=$ $\sqrt{(\boldsymbol{x}-\boldsymbol{\mu})^{\mathrm{T}} \Sigma^{-1}(\boldsymbol{x}-\boldsymbol{\mu})}$ is the Mahalanobis distance, and $D_{\mathrm{th}, m}(\alpha)$ is a percentile threshold of the $m$-dimensional $\chi^{2}$ distribution.

$D_{\mathrm{E}_{\mathrm{u}}, \min }$ is the minimum Euclidean distance that must be kept between the robot and the worker. $\psi$ is the angle from the closest point $\boldsymbol{p}_{\mathrm{c}, j}$ to the mean of the distribution $\mathcal{N}_{\mathrm{u}}^{(t)}$ as shown in Fig. 3. The uninvadable region is expressed by a circle whose center is located at the mean of the distribution $\mathcal{N}_{\mathrm{u}}^{(t)}$ and whose radius is $D_{\mathrm{E}_{\mathrm{u}} \text {, min }}$. The prediction uncertainty of the uninvadable region is considered using Mahalanobis distance between the closest point $\boldsymbol{p}_{\mathrm{c}, j}$ of the link and the distribution $\mathcal{N}_{\mathrm{u}}^{(t)}$. Note that eq. (25) calculates the Mahalanobis distance between $\left(\boldsymbol{p}_{\mathrm{c}, j}(\boldsymbol{q}(t))+\boldsymbol{D}_{\mathrm{E}_{\mathrm{u}}}\right)$ and the distribution $\mathcal{N}_{\mathrm{u}}^{(t)}$ instead of calculating the Mahalanobis distance between $\boldsymbol{p}_{\mathrm{c}, j}$ and the circumference of the circle.

\section{Continuous approximation of the switching constraint}

In the optimization problem, eq. (23) is a discontinuous function on the border of the collaborative region when the collaborative region and the uninvadable region are close to each other. In this case, it is not easy to use the methods applicable to the discontinuous function because of the prediction uncertainty of each region. We address this problem by approximating a discontinuous constraint to two continuous 


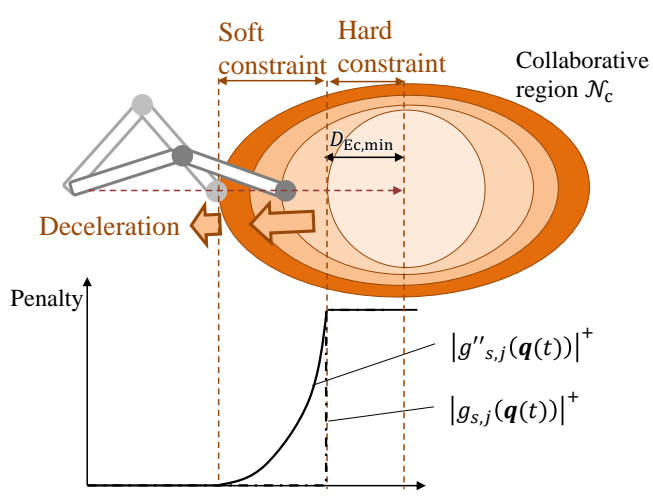

Fig. 4: Continuous approximation of a discontinuous constraint function using the Mahalanobis distance.

functions. First, the distance between $\boldsymbol{p}_{\mathrm{c}, j}$ and the collaborative region $\mathcal{N}_{\mathrm{c}}$ is calculated using the Mahalanobis distance. Then, the discontinuous constraint is approximated by two continuous functions using the Mahalanobis distance as follows:

$$
\begin{aligned}
& g_{s, j}^{\prime}(\boldsymbol{q}(t))=\left(1-e^{-\eta D_{\mathrm{M}_{\mathrm{c}}, j}(\boldsymbol{q}(t))}\right)\left\{D_{\mathrm{th}, m}(\alpha)-D_{\mathrm{M}_{\mathrm{u}}, j}(\boldsymbol{q}(t))\right\}, \\
& g_{s, j}^{\prime \prime}(\boldsymbol{q}(t))=e^{-\eta D_{\mathrm{M}_{\mathrm{c}}, j}(\boldsymbol{q}(t))}\left\{\left|\boldsymbol{v}_{j}(\boldsymbol{q}(t))\right|-v_{\max }\right\},
\end{aligned}
$$

where $D_{\mathrm{M}_{\mathrm{c}}, j}$ is the Mahalanobis distance between the Gaussian distribution $\mathcal{N}_{\mathrm{c}}$ and the closest point $\boldsymbol{p}_{\mathrm{c}, j}$ similar to the case of eqs. (24) through (26). $\eta$ is the weighting factor for the penalties eqs. (27) and (28).

Fig. 4 shows an example of the L1 penalty between the manipulator endpoint and the distribution $\mathcal{N}_{\mathrm{c}}$ when the endpoint is approaching the collaborative region. The penalty gradually increases due to the influence of the variance of the distribution $\mathcal{N}_{\mathrm{c}}$. The constraints function, eq. (16), is rewritten as

$$
\begin{aligned}
& G(\boldsymbol{q}(t), \boldsymbol{u}(t))=\rho \sum_{j=1}^{N_{j}}\left(\sum_{c=1}^{N_{c}}\left|g_{c, j}(\boldsymbol{q}(t), \boldsymbol{u}(t))\right|^{+}\right. \\
& \left.+\sum_{j=N_{s}}^{N_{j}}\left(\left|g_{s, j}^{\prime}(\boldsymbol{q}(t))\right|^{+}+\left|g_{s, j}^{\prime \prime}(\boldsymbol{q}(t))\right|^{+}\right)\right) .
\end{aligned}
$$

\section{Solver for the optimization problem}

The procedure for calculating the robot trajectory is shown in Algorithm 1. The collaborative region $\mathcal{N}_{\mathrm{c}}$, the uninvadable region $\mathcal{N}_{\mathrm{u}}$, the target arrival time $T_{\mathrm{a}}$, the target state $\boldsymbol{x}_{\mathrm{tgt}}$, the current state $\boldsymbol{q}_{\mathrm{cur}}$, and the trajectory calculated in the previous step $Q_{\text {pre }}$ are the inputs used for the calculation. The trajectory $Q$ is the output of this calculation and is used for robot control. Because the predicted positions of the worker corresponding to the uninvadable region $\mathcal{N}_{\mathrm{u}}$ are obtained for each sensor measurement cycle $\Delta t_{s}$, eqs. (10) and (12) are discretized in the sensor measurement cycle $\Delta t_{s}$.

After the optimal trajectory $\boldsymbol{Q}=\left(\boldsymbol{q}^{(k)}, \cdots, \boldsymbol{q}^{\left(k+T_{\mathrm{a}}\right)}\right)$ is calculated, whether each state $\boldsymbol{q}^{(k+l)}$ satisfies the constraints or not is evaluated. If a constraint is not satisfied, the weight factor $\rho$ is increased by the pre-defined factor $\beta$, and the optimization is executed again. Similar processing is repeated

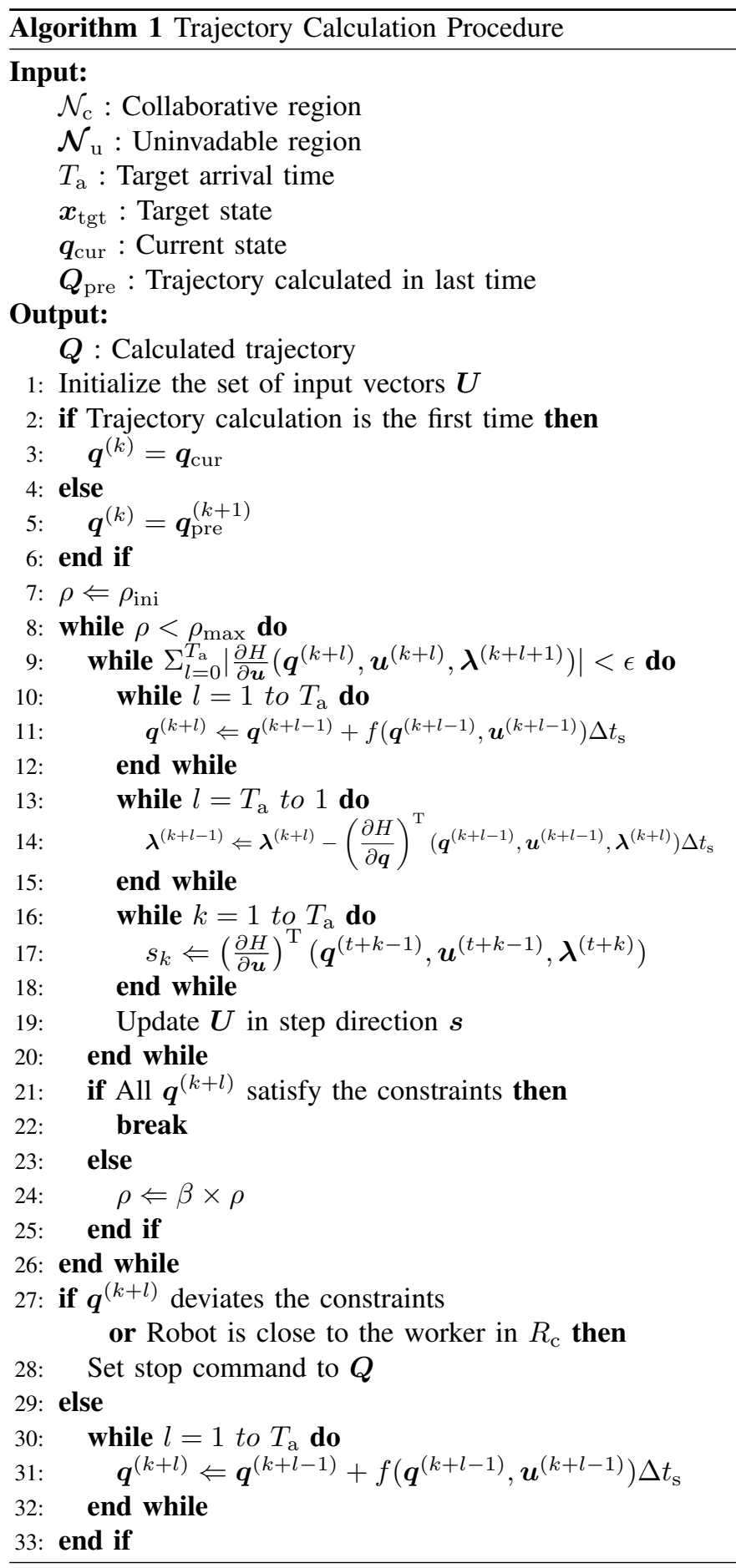

until the weight factor $\rho$ exceeds the maximum value $\rho_{\max }$. If all of the constraints are satisfied, the calculated optimal trajectory is adopted. If a trajectory that satisfies all of the constraints cannot be calculated, a command to stop the robot at its current position is issued instead of the calculated trajectory. This process allows the robot to safely stop when no optimal solution can be calculated due to the highly irregular movement of the worker. 


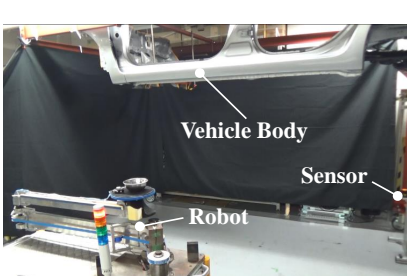

(a) Experimental environment

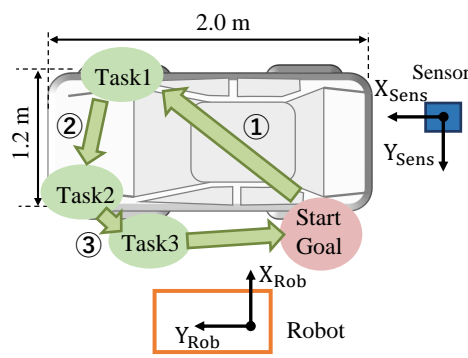

(b) Top view.
Fig. 5: Experimental setup.

TABLE I: Summary of the safe constraint parameters used in this experiment

\begin{tabular}{c|c} 
Parameters & Values \\
\hline \hline Radius of the collaborative region $D_{\mathrm{E}_{\mathrm{c}}, \min }$ & $0.6 \mathrm{~m}$ \\
\hline Radius of the uninvadable region $D_{\mathrm{E}_{\mathrm{u}}, \min }$ & $0.3 \mathrm{~m}$ \\
\hline Upper significance probability $\alpha$ & 0.05 \\
\hline Max TCP speed in the collaborative region $v_{\max }$ & $0.3 \mathrm{~m} / \mathrm{s}$ \\
\hline Max angular velocity $\left(\dot{\theta}_{1, \max }, \dot{\theta}_{2, \max }\right)$ & $(180,180) \mathrm{deg} / \mathrm{s}$ \\
\hline Max angular acceleration $\left(\ddot{\theta}_{1, \max }, \dot{\theta}_{2, \max }\right)$ & $(180,180) \mathrm{deg} / \mathrm{s}^{2}$ \\
\hline
\end{tabular}

\section{EXPERIMENT}

\section{A. Experimental setup}

In this paper, we evaluate the effectiveness of the proposed system in an environment similar to the actual automobile assembly process. Fig. 5(a) shows the experimental environment, and Fig. 5(b) shows the top view of the experimental setup. Fig. 5(b) shows the arrangement of the robot and the sensor and the assumed working positions and worker's pre-planned path. In this experiment, we use the collaborative robot system proposed in the previous study [32].

After the experiment starts, the worker attaches the parts to the overhead vehicle body at three working positions. The robot starts delivering the parts and the tool to the worker each time when the worker is predicted to reach each working position (Task1, Task2, and Task3). After the worker picks up the parts and the tool from the robot, the robot returns to its initial configuration and waits until the next task is started. One cycle of the experiment is completed when the worker finishes all three tasks and moves to the goal position shown in Fig. 5(b). Although the experimental environment is the same as in the previous study [3], this paper assumes highrisk collision cases such as a worker approaching the robot in motion or another second worker coming into the workspace.

In these settings, we prepare a total of 70 cases: 60 cases for one worker working and 10 cases for two workers working according to the pre-determined movement patterns. In the two-workers cases, another worker passes in front of the robot while the main worker moves to the next working position. The parameters of the motion planner used in this experiment are summarized in Table I. These parameters are used for all of the subsequent experiments.

\section{B. Evaluation}

In this experiment, the proposed method (objectiveswitching method) is compared with the existing methods (dependent-objective method and multi-objective method) to show the effectiveness of the proposed method.

The dependent-objective method is based on the maximum allowable TCP speed proportional to the distance between the robot and the worker. This concept has been proposed by Zanchettin et al. in [20]. Since the worker's position could not be predicted without uncertainties in our system, we use the Mahalanobis distance instead of the Euclidean distance. In this method, the constraint function eq. (16) can be expressed as

$G(\boldsymbol{q}(t), \boldsymbol{u}(t))=\rho \sum_{j=1}^{N_{j}}\left(\sum_{c=1}^{N_{c}}\left|g_{c, j}(\boldsymbol{q}(t), \boldsymbol{u}(t))\right|^{+}+\left|g_{d, j}(\boldsymbol{q}(t))\right|^{+}\right)$

$g_{d, j}(\boldsymbol{q}(t))=\left|\boldsymbol{v}_{j}(\boldsymbol{q}(t))\right|-\frac{D_{\mathrm{M}_{\mathrm{u}}, j}(\boldsymbol{q}(t))}{D_{\mathrm{th}, m}(\alpha)} v_{\max }$

The multi-objective method was proposed in our previous research [3]. In this method, the constraint function eq. (16) is expressed as

$G(\boldsymbol{q}(t), \boldsymbol{u}(t))=\sum_{j=1}^{N_{j}}\left(\rho \sum_{c=1}^{N_{c}}\left|g_{c, j}(\boldsymbol{q}(t), \boldsymbol{u}(t))\right|^{+}+w\left|g_{m, j}(\boldsymbol{q}(t))\right|^{+}\right)$

$g_{m, j}(\boldsymbol{q}(t))=D_{\mathrm{th}, m}(\alpha)-D_{\mathrm{M}_{\mathrm{u}}, j}(\boldsymbol{q}(t))$

where $w$ is the weighting factor for the L1 penalty $\left|g_{m, j}(\boldsymbol{q}(t))\right|^{+}$.

To compare these three methods under the same condition, the experiment using the proposed method is first carried out on actual environments, then the other two methods are evaluated in simulation experiments using the same movement data of the human worker obtained in the actual experiment.

The safety and time-efficiency of the robot's motion are evaluated using the experimental results. When the robot's TCP speed exceeds the safe speed, the delivery operation carried out by the robot is considered safe as long as the distance between the robot and the human worker is larger than the safe distance. In this experiment, the safe speed and distance are selected as $0.3 \mathrm{~m} / \mathrm{s}$ and $0.3 \mathrm{~m}$ respectively based on the maxim torque of the robot and preliminary experiments. The time-efficiency is evaluated based on the arrival time difference in the collaborative region between the worker and the robot, that is, their target position. In this paper, we define this time difference as the waiting time.

\section{Experimental results}

Fig. 6 shows an example of experimental results of the delivery task when the object receiver and another worker existed in the workspace. Each figure of Fig. 6 consists of a snapshot of the workspace and the top view of the workspace with predicted worker's trajectory, the uninvadable region $R_{\mathrm{u}}$, and the collaborative region $R_{\mathrm{c}}$. The blue region on the right side of the figures indicates the uninvadable region of the main worker who performs the assembly work, and the green region indicates the uninvadable region of the second worker who passes through the workspace without performing 


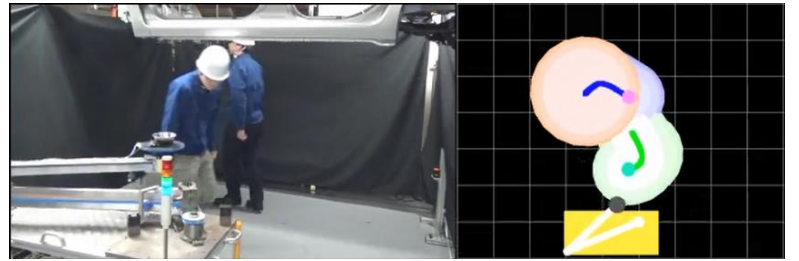

(a) The robot avoids contact with the passing worker.

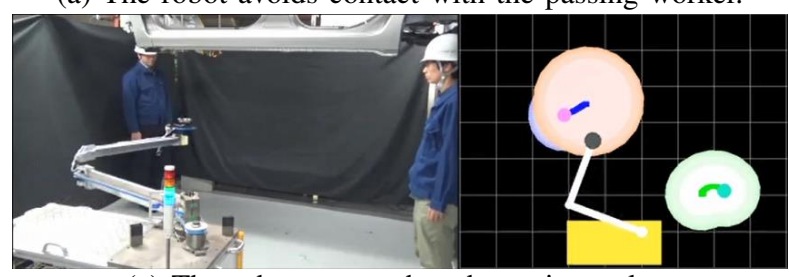

(c) The robot approaches the main worker.

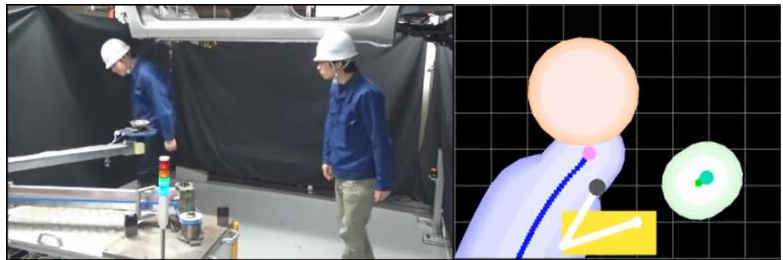

(b) The robot avoids contact with the main worker.

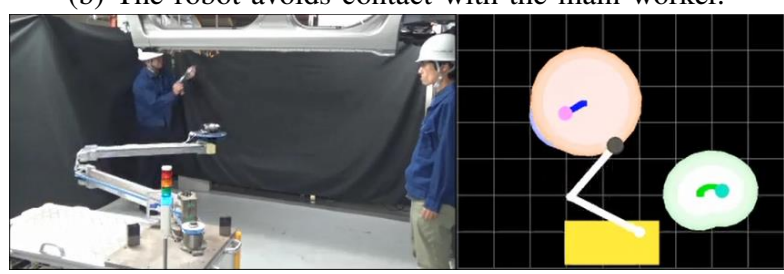

(d) The main worker starts the assembly task.

Fig. 6: Example of experimental results of the delivery task when the object receiver and another worker existed in the workspace. The full experiment is shown in the attached video.

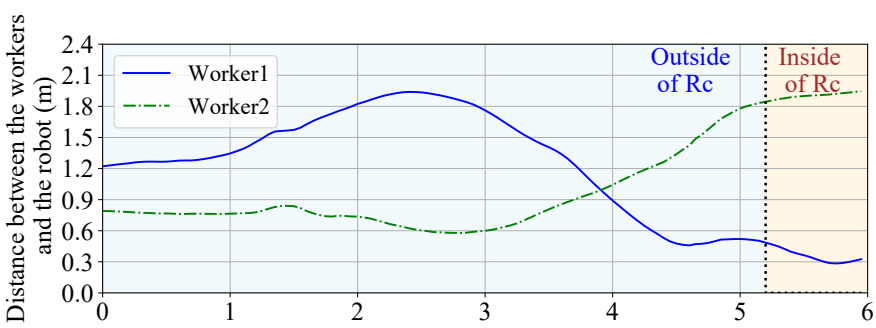

(a)

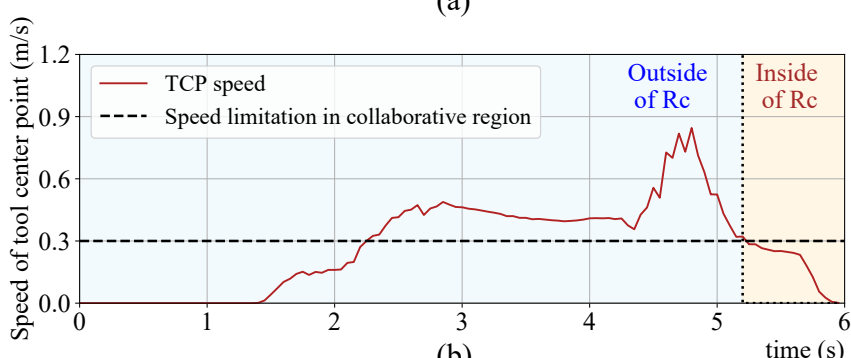

(b)

Fig. 7: The distances between the workers and the robot (a) and TCP speed (b)

assembly work. The robot first avoided the second worker who passed in front of the robot as shown in Fig. 6(a). Then the robot avoided the main worker who approached the robot as shown in Fig. 6(b). After avoiding the main worker, the robot approached the main worker in the collaborative region as shown in Fig. 6(c), and then the delivery task was completed as shown in Fig. 6(d).

Fig. 7 shows an example of the experimental results obtained during the experiment of Fig. 6. Fig. 7(a) shows the distances between the robot and each worker. You can see that the robot completed its delivery operation keeping the distance larger than the safe distance of $0.3 \mathrm{~m}$ when it was located outside of the collaborative region $R_{\mathrm{c}}$ as shown in the figure. Fig. 7(b) shows the TCP speed during the experiment. You can see that the robot TCP speed was reduced to the safe speed limit of $0.3 \mathrm{~m} / \mathrm{s}$ when the robot entered the collaborative region $R_{\mathrm{c}}$. In the collaborative region, the robot TCP speed was less than the speed limit.

1) Success rate of safe approaching: Table II summarizes the experimental results for each motion strategy. You can see that the proposed objective-switching method and the dependent-objective method could generate safe trajectories in all cases. On the other hand, the safe approaching could not be realized in some cases when multi-objective optimization was applied, because the multi-objective optimization can not simultaneously treat the contradictory constraints for the safe approach and the collision avoidance.

When the weight $\mathrm{w}$ was selected small, collisions occurred in 8 cases and excessive high-speed movement in close proximity to the worker occurred in 6 cases. This is due to the fact that reaching the target position has taken precedence over avoiding contact with the workers. When the weight $w$ was selected large, excessive high-speed movement in the close proximity occurred in 3 cases, and the computational trajectory diverged in 15 cases. This is because the conflicting objectives of reaching the target position and avoiding the collision could not be achieved at the same time when the uninvadable region $R_{\mathrm{u}}$ is located between the current robot pose and the collaborative region $R_{\mathrm{c}}$.

2) Efficiency of the robot delivery task: Fig. 8 shows the box plot of the waiting time obtained in each case. You can see that the waiting time of the proposed objective-switching method is shorter than that of the dependent-objective method. Although the waiting time of the multi-objective method with the small weight $w$ is the shortest, as mentioned above, many cases of the multi-objective method could not achieve the safe operation. The proposed method has a significant advantage compared to the multi-objective method in terms of safety.

We statistically evaluated the waiting time to know whether there was a significant difference between the proposed objective-switching method and the dependent-objective method. Both showed excellent performance in terms of safety. Since the obtained results are non-parametric groups and correspond to each other, we selected the Wilcoxon rank sum test. After testing the two groups, the p-value was obtained as 
TABLE II: Success rate of the robot delivery motion for each method from the viewpoint of safety

\begin{tabular}{c|c|c|c|c} 
Method & Total success rate & Failure: Collision & Failure: Excessive speed & Failure: Divergence \\
\hline \hline Objective-switching method & $70 / 70(100 \%)$ & $0 / 70$ & $0 / 70$ & $0 / 70$ \\
\hline Dependent-objective method & $70 / 70(100 \%)$ & $0 / 70$ & $0 / 70$ & $0 / 70$ \\
\hline Multi-objective method (w is low) & $56 / 70(80.0 \%)$ & $8 / 70(11.4 \%)$ & $6 / 70(8.6 \%)$ & $0 / 70$ \\
\hline Multi-objective method (w is high) & $52 / 70(74.3 \%)$ & $0 / 70$ & $3 / 70(4.3 \%)$ & $15 / 70(21.4 \%)$ \\
\hline
\end{tabular}

\section{REFERENCES}

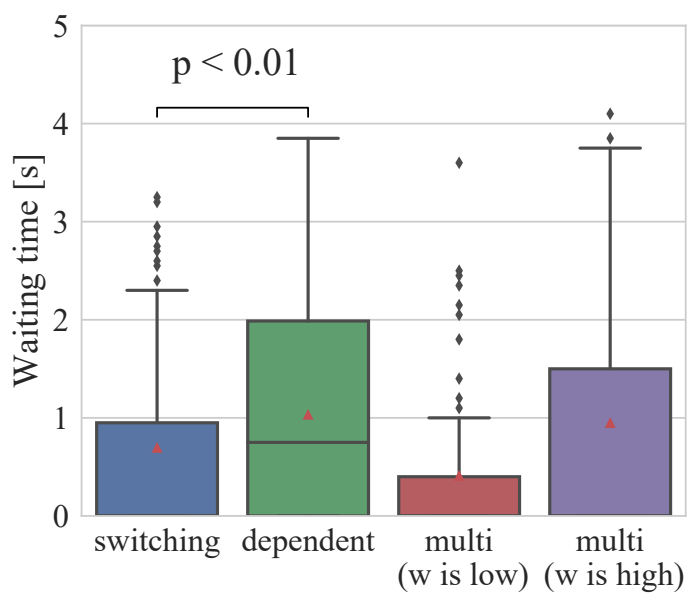

Fig. 8: Summary of the waiting time for all results of each method

$p=8.89 \times 10^{-5}<0.01$. Therefore, we statistically confirmed the significant difference in efficiency. The conducted experimental results showed that the proposed method has the best performance judging from both safety and efficiency points of view.

\section{CONCLUSION}

In this paper, we proposed the motion planning that explicitly integrated a strategy for safe approaching into the collision-free trajectory planning between the workers and the robot. The proposed method switches the objective functions of avoidance and approaching to the worker based on the current robot pose to satisfy the safety constraints. This strategy was integrated with prediction-based motion planning to achieve efficient robot movement considering the predicted worker's future movements and reliable collision avoidance based on the uncertainty of predictions. The problem of possible chattering near the boundary of the region $R_{\mathrm{c}}$, which is inherent to the switching of the objective functions, was solved by continuous approximation of the boundary based on the prediction uncertainty of the worker's position. The proposed scheme was implemented in the experimental system for the collaborative assembly scenario to evaluate the effectiveness of the proposed system. The results showed that the proposed system can simultaneously enhance time-efficiency and worker safety in considerably irregular and risky situations. We believe that the proposed strategy can solve essential issues in the motion planning of robots that cooperate with humans and can lead to better collaboration between humans and robots in various fields.
[1] V. Villani, F. Pini, F. Leali, C. Secchi, "Survey on human-robot collaboration in industrial settings: Safety, intuitive interfaces and applications", Mechatronics, vol. 55, pp. 248-266, 2018.

[2] ISO 10218-1:2011 - Robots And robotic devices - Safety requirements for industrial robots - Part 1: robots. ISO;2011.

[3] A. Kanazawa, J. Kinugawa, K. Kosuge, "Adaptive motion planning for a collaborative robot based on prediction uncertainty to enhance human safety and work efficiency", IEEE Transactions on Robotics, vol. 35, no. 4, pp. 817-832, 2019.

[4] S. Li, J.A. Shah, "Safe and efficient high dimensional motion planning in space-time with time parameterized prediction", In Proceedings of International Conference on Robotics and Automation (ICRA), pp. 5012$5018,2019$.

[5] K. Wei, B. Ren, "A method on dynamic path planning for robotic manipulator autonomous obstacle avoidance based on an improved RRT algorithm", Sensors, vol. 18, no. 2, pp. 571-585, 2018.

[6] N. Ratliff, M. Zucker, J. A. Bagnell and S. Srinivasa, "CHOMP: Gradient Optimization Techniques for Efficient Motion Planning”, In Proceedings of International Conference on Robotics and Automation (ICRA), pp. 489-494, 2009.

[7] M. Kalakrishnan, S. Chitta, E. Theodorou, P. Pastor and S. Schaal, "STOMP: Stochastic Trajectory Optimization for Motion Planning", In Proceedings of International Conference on Robotics and Automation (ICRA), pp. 4569-4574, 2011.

[8] C. Park, J. Pan and D. Manocha, "ITOMP: Incremental Trajectory Optimization for Real-Time Replanning in Dynamic Environments", In Proceedings of International Conference on Automated Planning and Scheduling (ICAPS), pp. 207-215, 2012.

[9] J. Schulman, J. Ho, A. X. Lee, I. Awwal, H. Bradlow and P. Abbeel, "Finding Locally Optimal, Collision-Free Trajectories with Sequential Convex Optimization", In Robotics: Science and Systems, vol. 9, no. 1, pp. 1-10, 2013.

[10] J. J. Kim and J. J. Lee, "Trajectory Optimization with Particle Swarm Optimization for Manipulator Motion Planning", IEEE Transactions on Industrial Informatics, vol. 11, no. 3, pp. 620-631, 2015.

[11] O. Andersson, M. Wzorek, P. Rudol, P. Doherty, "Model-predictive control with stochastic collision avoidance using bayesian policy optimization", In Proceedings of International Conference on Robotics and Automation (ICRA), pp. 4597-4604, 2016.

[12] T. Seyde, J. Carius, R. Grandia, F. Farshidian, M. Hutter, "Locomotion Planning through a Hybrid Bayesian Trajectory Optimization", In Proceedings of International Conference on Robotics and Automation (ICRA), pp.5544-5550, 2019.

[13] R. Hayne, R. Luo and D. Berenson, "Considering avoidance and consistency in motion planning for human-robot manipulation in a shared workspace", In Proceedings of International Conference on Robotics and Automation (ICRA), pp.3948-3954, 2016.

[14] J. H. Chen, K. T. Song, "Collision-free motion planning for humanrobot collaborative safety under Cartesian constraint",In Proceedings of International Conference on Robotics and Automation (ICRA), pp. 1-7, 2018.

[15] N.M. Ceriani, A.M. Zanchettin, P. Rocco, A. Stolt and A. Robertsson, "Reactive task adaptation based on hierarchical constraints classification for safe industrial robots", IEEE/ASME Transactions on Mechatronics, vol. 20 no. 6, pp.2935-2949, 2015.

[16] C. Liu, M. Tomizuka, "Algorithmic safety measures for intelligent industrial co-robots", In Proceedings of International Conference on Robotics and Automation (ICRA), pp. 3095-3102, 2016.

[17] D. Kuli, E. A. Croft, "Safe planning for human- robot interaction", Journal of Robotic Systems, vol. 22, no. 7, pp. 383-396, 2005.

[18] R. A. Rojas, M. A. R. Garcia, E. Wehrle, R. Vidoni, "A variational approach to minimum-jerk trajectories for psychological safety in collaborative assembly stations", IEEE Robotics and Automation Letters, vol. 4, no. 2, pp. 823-829, 2019. 
[19] R. Weitschat, H. Aschemann, "Safe and Efficient Human-Robot Collaboration Part II: Optimal Generalized Human-in-the-Loop Real-Time Motion Generation", IEEE Robotics and Automation Letters, vol. 3, no. 4, pp. 3781-3788, 2018.

[20] A. M. Zanchettin, N. M. Ceriani, P. Rocco, H. Ding, B. Matthias, "Safety in human-robot collaborative manufacturing environments: Metrics and control", IEEE Transactions on Automation Science and Engineering, vol. 13, no. 2, pp. 882-893, 2015.

[21] J. Mainprice and D. Berenson, "Human-Robot Collaborative Manipulation Planning Using Early Prediction of Human Motion", In Proceedings of International Conference on Intelligent Robots and Systems (IROS), pp. 299-306, 2013.

[22] D. Fridovich-Keil, A. Bajcsy, J. F. Fisac, S. L. Herbert, S. Wang, A. D. Dragan, C. J. Tomlin, "Confidence-aware motion prediction for real-time collision avoidance". The International Journal of Robotics Research, vol. 39, no.2-3, pp.250-265, 2020.

[23] J. S. Park, C. Park and D. Manocha, "Intention-Aware Motion Planning Using Learning Based Human Motion Prediction. In Robotics: Science and Systems, 2017.

[24] Y. Wang, Y. Sheng, J. Wang, W. Zhang, "Optimal collision-free robot trajectory generation based on time series prediction of human motion", IEEE Robotics and Automation Letters, vol. 3, no. 1, pp. 226-233, 2017.

[25] V. V. Unhelkar, P. A. Lasota, Q. Tyroller, R. D. Buhai, L. Marceau, B. Deml and J. A. Shah, "Human-Aware Robotic Assistant for Collaborative Assembly: Integrating Human Motion Prediction with Planning in Time", IEEE Robotics and Automation Letters, vol. 3, no. 3, pp. 2394-240, 2018.

[26] K. P. Hawkins, N. Vo, S. Bansal, A. F. Bobick, "Probabilistic human action prediction and wait-sensitive planning for responsive humanrobot collaboration", International Conference on Humanoid Robots (Humanoids), pp. 499-506, 2013.

[27] G. Maeda, M. Ewerton, G. Neumann, R. Lioutikov, J. Peters, "Phase estimation for fast action recognition and trajectory generation in humanrobot collaboration", The International Journal of Robotics Research, vol. 36 no.13-14, pp. 1579-1594, 2017.

[28] C. Pérez-D' Arpino, J. A. Shah, "Fast target prediction of human reaching motion for cooperative human-robot manipulation tasks using time series classification", In Proceedings of International Conference on Robotics and Automation (ICRA), pp. 6175-6182, 2015.

[29] A. Kanazawa, J. Kinugawa, K. Kosuge, "Incremental Learning of Spatial-Temporal Features in Human Motion Patterns with Mixture Model for Planning Motion of a Collaborative Robot in Assembly Lines", In Proceedings of International Conference on Robotics and Automation (ICRA), pp. 7858-7864, 2019.

[30] A. E. Bryson, "Applied optimal control: optimization, estimation and control", Routledge, 2018.

[31] T. Ohtsuka, "A Continuation/GMRES Method for Fast Computation of Nonlinear Receding Horizon Control", Automatica, vol. 40 no. 4 , pp. 563-574, 2004.

[32] J. Kinugawa, Y. Sugahara, and K. Kosuge, "Co-worker Robot," Acta Polytechnica Hungarica, vol. 13, no. 1, pp. 209-221, 2016.
Jun Kinugawa

PLACE

PHOTO

HERE

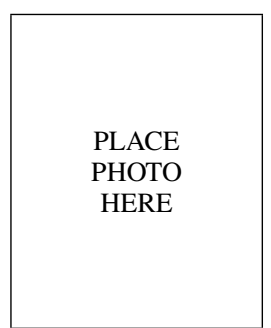

Kazuhiro Kosuge

Akira Kanazawa

PLACE

PHOTO

HERE 